\title{
Life-time estrogen exposure and cognitive functioning in later life
}

Running Title: Estrogen exposure and cognitive function

*Joanne Ryan ${ }^{1,2,3}$, Isabelle Carrière ${ }^{1}$, Jacqueline Scali ${ }^{1}$, Karen Ritchie ${ }^{1}$, Marie-Laure Ancelin ${ }^{1}$.

${ }^{1}$ Inserm, U888, Montpellier, F-34093 France ;

${ }^{2}$ Univ Montpellier1, Montpellier, F-34000 France

${ }^{3}$ Department of Psychiatry, The University of Melbourne, Melboure, Australia

*Corresponding Author:

Inserm U888, Nervous System Pathologies: Epidemiological and Clinical Research. Hôpital La Colombière, 39 av. Ch. Flahault, BP 34493, 34093 Montpellier Cedex 5, France.

Tel: +33 499614 562, Fax: +33 499614579 . Email: joanne.ryan@inserm.fr, 


\section{SUMMARY}

Context: While recent studies suggest that exogenous estrogen treatment could help reduce age-related cognitive decline and delay the onset of dementia, this has not been found consistently. Few studies have considered the influence of life-time estrogen exposure which may have an independent effect on cognition and/or modulate treatment response.

Objective: The aim of this study was to examine whether factors related to estrogen exposure across the lifetime were associated with cognitive function in postmenopausal women.

Design: A battery of cognitive tests were administered at baseline and at two and four years of follow-up to evaluate cognitive performance among a population based cohort of 996 French women aged 65 years or older, who were recruited as part of the ESPRIT Study. Detailed reproductive histories were also obtained. Logistic regression models, controlling for an extensive range of potential confounding factors, were generated to determine whether hormone-related factors across a woman's lifetime were associated with poor cognitive performance in later life.

Results: Age at first menses was negatively associated with performance on the tasks of visual memory and psychomotor speed, while a longer reproductive period was associated with better verbal fluency. Likewise, women who had their first child at a young age performed significantly worse on each of these tasks, as well as on a measure of global cognitive function. The results also suggest that current hormone therapy may be beneficial for a number of cognitive domains, however in multivariate analysis, women performed significantly better on the task of visual memory only. In contrast, in analysis adjusted for baseline cognitive performance and a range of other factors, none of the reproductive variables were associated with a decline in cognitive performance or the incidence of dementia during the four year follow-up period.

Conclusions: In addition to hormone therapy, certain hormone-related events across the lifetime are also associated with cognitive functioning in later life. They were not observed in this study to modulate dementia risk; however this should be verified over a longer follow-up period. Further studies will also be required to determine whether lifetime hormonal exposure may modify women's response to hormone therapy after the menopause.

Key words: Cognition, Dementia, Lifetime Exposure, Estrogen, Hormone Therapy 


\section{INTRODUCTION}

Epidemiological studies suggest that elderly women have the highest prevalence of both dementia and more specifically Alzheimer's disease (AD) (Gao et al., 1998; Andersen et al., 1999), and that reduced levels of estradiol that accompany the postmenopausal period, may be partly responsible. Estrogen affects the structure and function of the nervous system and appears to be involved in brain maturation and memory processing (Beh1, 2002). Estrogen has been shown to have a positive influence on brain neurotransmitter levels and the identification of estrogen receptors in the brain suggests they mediate some of the hormone's cognitive effects (see for review (Ancelin and Ritchie, 2005)). The potential for estrogen to reduce agerelated cognitive problems and delay the onset of more severe cognitive deficits such as dementia, is therefore of strong interest. However, despite relatively clear evidence for a biological link between estrogen and cognitive function, a protective effect has not been consistently demonstrated in clinical studies (see for review (Sherwin and Henry, 2008)).

The vast amount of work in this field has come from studies of hormone therapy (HT), which have investigated the potential therapeutic role of estradiol, with or without progesterone, on cognitive function (see for review (Ryan et al., 2008b; Sherwin and Henry, 2008)). Meta-analyses of observational studies have reported predominantly positive effects of HT on cognitive function, most notably with respect to verbal memory, and a diminished risk of AD has also been reported in users of HT compared to non-users (Yaffe et al., 1998; Leblanc et al., 2001; Maki and Hogervorst, 2003). However, the "gold-standard" randomised controlled-trials (RCTs) have been mixed and the Women's Health Initiative Memory Study (WHIMS), an ancillary study to the much publicised WHI, actually found that women using HT had an increased risk of developing all cause-dementia (Shumaker et al., 2003). In addition, women using treatment were not at an increased risk of mild cognitive impairment (MCI) and the risk of overall cognitive dysfunction was not modified compared with women taking placebo (Rapp et al., 2003; Shumaker et al., 2003). Subsequent criticisms of this study have pointed out that the female participants were aged at least 65 years and therefore this is not truly representative on the usual clinical situation, where women often seek treatment around the time of the menopause. These women were furthermore treated with a particular type of HT, an orally administered conjugated equine estrogen compound that is used widely in the USA but not necessarily elsewhere in the world. Treatment with transdermal estradiol compounds may produce quite different results (Sherwin and Henry, 2008). 
The current inconsistencies in the literature may therefore result from differences in study designs; notably types of HT, age of the populations involved and differences in the type and measurement of the cognitive functions examined. While there is evidence to suggest that HT may preferentially benefit shortterm verbal memory (Hogervorst et al., 2002b; Sherwin, 2003), positive associations have also been reported with other less extensively studied cognitive domains, including verbal fluency (Grodstein et al., 2000), visual memory (Resnick et al., 1997) and psychomotor speed (MacLennan et al., 2006). It is also possible that rather than focusing solely on HT, it may be important to consider other hormonal factors that could contribute to late-life cognitive function, such as other forms of exogenous treatment (principally oral contraceptives) and factors which influence endogenous estrogen exposure across the lifetime.

Data from epidemiological studies provide some indication of an association between increased lifetime endogenous exposure and decreased risk of poor cognitive function (Smith et al., 1999; Lebrun et al., 2005; Rasgon et al., 2005), cognitive decline (McLay et al., 2003) or AD (Paganini-Hill and Henderson, 1994; Ptok et al., 2002; Colucci et al., 2006). However studies have tended to focus on only a small number of reproductive events, principally age at menopause (Dunkin et al., 2005; Lebrun et al., 2005) or considering in addition the timing of first menses (Paganini-Hill and Henderson, 1994; Geerlings et al., 2001; Low et al., 2005). Of the studies which have looked at a number of reproductive factors (age at menarche and menopause, as well as parity) (Rasgon et al., 2005; Colucci et al., 2006), including one of the scant longitudinal studies to be undertaken in this area (McLay et al., 2003), none of these have examined which specific areas of cognitive functioning are most susceptible to hormone exposure.

The aim of this study was to determine whether factors that influence both endogenous and exogenous hormone exposure across the lifetime, are associated with cognitive function in later life or the decline in cognitive function over a 4-year period. In addition to verbal memory, other less extensively studied cognitive domains were also investigated. In this study, we considered the multiple competing causes of cognitive decline in the elderly and the incidence of dementia was also evaluated, to determine whether hormonal factors can modify dementia risk. We hypothesised that increased endogenous estrogen exposure across the lifetime or current HT would be associated with better cognitive function in later life. 


\section{METHODS}

\section{Participants}

Details regarding the design of the ESPRIT study and participant recruitment have been published in detail (Ritchie et al., 2004). Participants were recruited over a two-year period from 1999 to 2001, by random selection from the electoral rolls in Montpellier, France. Eligible individuals, who were at least 65 years of age and non-institutionalised, were invited to participate and provided written informed consent. Participants were administered a number of standardised questionnaires by trained staff and underwent clinical examinations at baseline. Follow-up takes place every two years and is ongoing, but the data described here concern the first four years of the study. Ethics approval for the ESPRIT study was granted by the Ethics Committee of the University Hospital of Kremlin-Bicêtre (France) and all procedures were carried out with the adequate understanding and written consent of the participants.

A total of 1313 women were initially recruited to the ESPRIT cohort, however this current analysis is based on 996 of these women. Women were excluded from this analysis if they were diagnosed with possible or probable dementia at inclusion $(n=36)$, if they died or were otherwise lost to follow-up during the four year study period $(n=102)$, if they had incomplete data relating to the cognitive tests administered at baseline or follow-up $(\mathrm{n}=78)$ or if they were missing at least some of the data concerning the covariates included in the multivariate analysis $(\mathrm{n}=101)$.

\section{Cognitive Assessment}

A battery of cognitive tests was administered by trained staff and assessed different areas of cognitive functioning at baseline and each year of follow-up. Verbal memory was examined with the 5 -word Test of Dubois, with both immediate and delayed recall tasks (Dubois, 2001) and the total number of words correctly recalled was calculated (maximum 10). Isaacs Set Test (Isaacs and Kennie, 1973) provided a measure of verbal fluency or semantic access which is sensitive to changes in both frontal and temporal areas. Participants were asked to generate as many words as possible within a given semantic category (animals, colors, fruits and cities) and their total score was the sum of the number of words generated in each category within 30 seconds. Benton's Visual Retention Test (BVRT) (Benton, 1965) assessed visual memory; psychomotor speed and executive function were assessed with the Trail Making Tests A and B respectively (TMA and TMB) (Reitan, 1965) and the Mini Mental State Examination (MMSE) (Folstein et 
al., 1975) was used as a global measure of cognitive function. All of the cognitive tests were administered at baseline, and during wave 1 and 2 of the follow-up, except the Trail Making tasks A and B which not given in wave 1 . Consequently, the analysis based on these tasks involves 181 fewer participants. We defined low cognitive performance as scoring in the lowest quintile for each cognitive test. Over the follow-up period, a substantial decline in cognitive function was defined as being in the lowest quintile of the difference between baseline score and either of the follow-up visits.

At baseline and each follow-up interview, all participants were examined by a neurologist and a standard clinical protocol was used for the diagnoses and classification of dementia based on DSM-IV criteria (American Psychiatric Association, 1994). All incident cases were further validated by a group of neurological experts and when dementia was diagnosed, the date of onset was recorded as the date of the follow-up interview.

\section{Reproductive Characteristics}

From work undertaken most notably in the field of breast cancer (Clavel-Chapelon, 2002), factors that are thought to influence estrogen exposure across the lifetime have been identified. An increasing number of reproductive years, resulting from a younger age at menarche and/or an older age at menopause, indicates a higher lifetime endogenous estrogen exposure (Clavel-Chapelon, 2002). On the other hand, estrogen exposure is expected to decrease with parity and an earlier age at child birth (Kalache et al., 1993). The type of menopause is also expected to influence estrogen exposure, as surgical menopause results in a more dramatic decline in estrogen levels than natural menopause (Henderson and Sherwin, 2007). A specific questionnaire concerning reproductive lifetime events and hormonal exposure was administered as part of a general clinical examination (Ryan et al., 2008a), requesting information on all of the factors mentioned above. Information was also recorded on the participant's use of exogenous hormonal treatment, including oral contraceptives and HT, and the duration of such treatment.

\section{Other variables}

At inclusion, information was obtained on socio-demographic and lifestyle characteristics, as well as general health. Based on findings reported in the literature, the variables described below were considered as potential covariates in our analysis, because they may influence cognitive performance and were potentially 
linked to the use of HT or other reproductive markers. Participants were classified as disabled if they were unable to complete at least two tasks from either the Instrumental Activities of Daily Living (IADL) (Lawton and Brody, 1969) or the Activities of Daily Living (ADL) (Katz et al., 1963) scales. Depressive symptoms were assessed using the Centre for Epidemiology Studies Depression Scale (CES-D) (Radloff, 1977) and women who scored 16 or more on the questionnaire were classified as depressed. Regular smoking was defined as smoking at least 10 packets per year. High alcohol consumption was at least 24 grams of alcohol each day and high caffeine intake three or more cups of coffee a day (or equivalently combined with tea intake, with each cup of tea having approximately half the caffeine intake) (Ritchie et al., 2007). Body mass index (BMI) was calculated as weight $(\mathrm{kg})$ divided by height $\left(\right.$ metres $\left.^{2}\right)$. Based on standard criteria (National Heart, 1998) women with a very low BMI were labelled as underweight $(\mathrm{BMI} \leq 18)$, those in the highest range, as obese (BMI $\geq 30)$ and all others were considered as having "normal" BMI.

Participants provided fasting blood samples and responded to detailed medical questionnaires. Information was obtained on history of vascular diseases (including angina pectoris, myocardial infarction, stroke, cardiovascular surgery, bradycardia or palpitations), other chronic illnesses (asthma, diabetes (fasting glucose $\geq 7.2 \mathrm{mmol} / 1$ or reported treatment), hypercholesterolemia (total cholesterol $\geq 6.2 \mathrm{mmol} / 1$ ), hypertension (resting blood pressure $\geq 160 / 95 \mathrm{~mm} \mathrm{Hg}$ or treated) and thyroid problems), use of anticholinergic medication (Ancelin et al., 2006) and diagnoses of cancer within the last 2 years. Participants were classified as having a chronic disease if they suffered from one or more of these illnesses. Apolipoprotein E genotyping was carried out from blood samples and participants were identified as either carriers or not of the ApoE-ع4 allele (Dufouil et al., 2005). Current use of medications was validated either by presentation of the prescription or the medication itself and the type of medication was noted according to the World Health Organisation's ATC classification (WHO., 2000).

\section{Statistical Analysis}

Two-tailed chi-squared tests were used to determine the bivariate associations between baseline characteristics and cognitive function. Logistic models were generated, initially adjusting for age and education only, to examine the association between poor cognitive performance at inclusion and lifetime reproductive characteristics. The hormonal characteristics which were associated with cognitive performance at the $20 \%$ significance level $(p<0.2)$, were then considered simultaneously in logistic models adjusted for 
age, education level, marital status, depressive symptoms, high caffeine intake, physical incapacities and comorbidity. These covariates were selected a priori based on the literature (Ritchie et al., 2007), and also from chi-squared analyses indicating the variables which were related to cognitive performance. The final multivariate models (one for each cognitive domain) contained the hormonal variables that remained significantly associated with cognitive function after inclusion of all of the potential confounders.

Multivariate logistic analysis was also used to determine whether baseline hormone-related factors were associated with the risk of cognitive decline over the four year follow-up, while adjusting for the potential confounding factors and their baseline cognitive scores. In addition, random-effect models were used to analyse the associations between the hormone factors and cognitive scores taken as continuous variables. Due to their skewed distribution, the Trail Making tests A and B were log transformed, while the Benton and MMSE variables were transformed using (15 minus Benton Score) ${ }^{1 / 2}$ and (30 minus MMSE $\mathrm{Score}^{1 / 2}$ respectively (Ritchie et al., 2007). The scores on the word recall task could not be normalised. Each of the models included an interaction term between time and the relevant hormone variable, which represented the annual modification these variables had on cognitive function. Finally, Cox proportional hazards models with delayed entry were developed to determine which reproductive factors were associated with the incidence of dementia during the follow-up period. To avoid the problem of non-proportionality in dementia risk with age, age itself was taken as the basic time scale and birth as the time origin (Commenges et al., 1998).

There was no indication of collinearity between any of the covariates in the different models and no statistically significant interaction terms. SAS version 9.1 (SAS Institute, Inc., Cary, North Carolina) was used for all of the statistical analysis and the significance level was $\mathrm{p}<0.05$. 


\section{RESULTS}

\section{Baseline characteristics of participants}

The baseline characteristics of the women in this study are shown in Table 1 . The women ranged from 65 to 94 years of age, with a mean of 73 years. Just over one-quarter of the participants had a tertiary education and the majority were either married or had a partner. Only a small proportion of the women had physical incapacities; however the rate of comorbidity (37.7\%) and depressive symptoms (34.5\%) was relatively high. The mean age at menopause was approximately 50 and in over $80 \%$ of cases this was a natural menopause. Almost a fifth of all women reported that they used oral contraceptives during their reproductive years, and $12 \%$ were nulliparous. Current HT use was relatively frequent given the age of the women.

Compared to the entire ESPRIT cohort, the women included in this analysis were more likely to have a higher education $\left(\chi^{2}=14.1, \mathrm{df}=1, \mathrm{p}<0.001\right)$ and to be younger $(\mathrm{t}=2.8, \mathrm{df}=1, \mathrm{p}=0.005)$. However, they were less likely to be depressed $\left(\chi^{2}=12.78, \mathrm{df}=1, \mathrm{p}<0.001\right)$, have comorbidity $\left(\chi^{2}=8.8, \mathrm{df}=1, \mathrm{p}=0.003\right)$ and incapacities $\left(\chi^{2}=70.7, \mathrm{df}=1, \mathrm{p}<0.001\right)$ and were significantly less likely to perform poorly on the cognitive tests $(\mathrm{df}=1, \mathrm{p}<0.001$ for all cognitive tests). All of the other socio-demographic, health and lifestyle characteristics, as well as the hormone-related characteristics which were examined, did not differ between the women included in this analysis and those that were not.

\section{Association between hormone-related factors and cognitive function at baseline}

After initial age and education adjusted logistic analysis (Supplementary Table 1), the hormone variables which appeared to be associated with cognitive function at the $20 \%$ significance level were then combined together in multivariate adjusted models. The hormone-related factors which remained significant independent predictors of poor performance on each of the cognitive tests are shown in Table 2.

Age at the birth of the first child was the only reproductive factor which remained significantly associated with global cognitive performance (MMSE) after multivariate adjustment. Women who were between the ages of 21 and 29 when their first child was born had better cognitive performance compared to women who gave birth at a younger age. Older first time mothers ( $\geq 30$ years) were not significantly better or worse off than young mothers. A similar positive association with age at childbirth was seen with the Isaacs Set Test, but in this case, women who had their first child later in life also had better verbal fluency. In addition, there was a strong positive association between the length of the reproductive period and the score 
on Isaacs Set Test. For every extra year between the age at first menses and the age at menopause, the risk of a low cognitive score decreased by $4 \%$.

The multivariate models for poor visual memory (BVRT) and psychomotor speed (TMA) were very similar, with age at first menses and age at the birth of the first child remaining significant in the final adjusted models. In both models, a later age at first menses increased the risk of poor cognitive performance, while women who had their first child before the age of 21 , also had an increased risk compared to first time mothers who were aged between 21 and 29 years old. In addition, current but not past HT was associated with higher cognitive function, but only reached statistical significance in the case of visual memory $(p=0.03)$. Likewise, there was a strong trend for current HT having a beneficial effect on their performance on the word recall task $(p=0.08)$, as well as the measure of executive function (TMB) $(p=0.09)$. In both cases however, this failed to reach the $5 \%$ significance level.

The number of children a woman had did not appear to be associated with modified performance on any of the cognitive tasks; however nulliparity was associated with a significantly better visual memory in the initial models before multivariate adjustment $(\mathrm{OR}=0.56,95 \% \mathrm{CI}: 0.32-0.98)$. Neither oral contraceptive use nor surgical menopause, were associated with performance on any of the cognitive tests.

\section{Hormone-related factors and change in cognitive function over time}

We then examined whether these hormone-related factors could be associated with alterations in cognitive function over the follow-up period. Once again, in the first step we used logistic analysis to determine risk factors for a decline in cognitive function while adjusting for age, education level and the baseline cognitive score (Table 3). Overall, the results indicate that these reproductive factors were associated with a minimal longitudinal change in cognitive score. The only significant associations were a beneficial effect of a later age at menopause $(\mathrm{OR}=0.97,95 \% \mathrm{CI}: 0.94-1.00)$ or similarly a longer reproductive period $(\mathrm{OR}=0.96,95 \%$ CI: 0.94-0.99), on the decline in executive function (TMB). These factors, in addition to those that were associated with cognitive decline at the $20 \%$ level, were thus retained for the multivariate analysis. However, after further adjustment for depression, marital status, comorbidity, physical incapacities and high caffeine intake, none of the reproductive factors were significant independent predictors of cognitive decline. This finding was further supported by the results of the random-effects mixed models, where cognitive decline was treated as a continuous variable (data not shown). 
With regard to dementia, a delayed-entry Proportional Hazards Cox model adjusted for education, failed to show a significant association between the use of HT at baseline and the incidence of dementia from all causes $(n=27)$ over the four year follow-up period $(p=0.93)$. None of the other hormonal factors either, were shown to influence dementia risk. 


\section{DISCUSSION}

The results of our study suggest that, in addition to HT, reproductive factors which influence endogenous estrogen exposure across the lifetime are associated with cognitive function in postmenopausal women and these hormone factors can have differential effects across cognitive domains.

\section{Endogenous Hormone Factors}

While different multivariate models were generated for each cognitive domain and a number of hormone events were examined, only three factors related to endogenous estrogen exposure, namely timing of the first menses, length of reproductive life and age at the birth of the first child, appeared to be associated with cognitive function.

An early menarche is associated with the early establishment of regulatory ovulatory cycles (Apter and Vihko, 1983) and with significantly higher estradiol levels in adolescents and young adults (MacMahon et al., 1982; Apter et al., 1989). In addition, age at menarche is widely established as a risk factor for hormone-dependent disorder such as breast cancer (Bernstein and Ross, 1993), however only a few studies have investigated its effect on later life cognitive function. A later age at menarche has been associated with an increased risk of cognitive impairment, based on a brief telephone cognitive screen to identify cases of dementia (Rasgon et al., 2005), as well as an increased risk of AD (Paganini-Hill and Henderson, 1994), although particular areas of cognitive function were not examined. We found that a later age at first menses was associated specifically, with lower performance in the areas of visuospatial memory (BVRT) and psychomotor speed (TMA).

The results of our study indicate that a later age at menopause or a longer reproductive period were associated with a significantly better verbal fluency; a finding which has not been demonstrated previously. Endogenous estrogen exposure is highest during a woman's reproductive life, which commences with the first menses and terminates at the menopause. Length of the reproductive period thus provides a simple estimate of a woman's lifetime estrogen exposure (Low et al., 2005). In agreement with other studies we found no association between length of reproductive life and verbal memory (Nappi et al., 1999; Henderson et al., 2003; Low et al., 2005), visual memory, attention or psychomotor speed (Nappi et al., 1999). We also found no association between the length of the reproductive period and global cognitive function, although others have reported that a later age at menopause was associated with better cognition (MMSE) (Lebrun et 
al., 2005). As these results were found in cross-sectional analysis, we cannot draw any conclusions about cause and effect. It is also possible, for example, that rather than age of menopause influencing cognitive function in older age, cognitive function at a younger age may in fact impact on the age at natural menopause. Somewhat surprisingly, a study by Kuh et al., actually found that higher cognitive scores during childhood, were associated with a later age at menopause (Kuh et al., 2005). Like the majority of other studies in this area, we have no data concerning the cognitive function of the women when they were younger, which may explain at least in part, the associations reported here.

In the initial models which were adjusted for age and education only (supplementary Table 1), we found that parity, but not the number of children was associated with poor visual memory. However after multivariate adjustment and the incorporation of several hormone factors into the one model (Table 2), parity no longer remained significant. On the other hand, among the parous women, those who had their first child at a young age $(\leq 20)$ were found to perform significantly worse on a number of cognitive tasks, even after multivariate adjustment. It has been reported that parous women may have shorter menstrual cycles and significantly lower levels of estradiol than nulliparous women (Bernstein et al., 1985; Dorgan et al., 1995) and these effects may be particularly evident after the first pregnancy (Bernstein et al., 1986; Musey et al., 1987). Thus, age at the birth of the first child appears to influence estrogen exposure. Data in the field of hormone-dependent breast cancer once again, support an association between breast cancer risk and age at childbirth, particularly for women who give birth before the age of 20 (Trapido, 1983; Britt et al., 2007). It is interesting to note that women who gave birth before the age of 21 have an increased risk of poor cognitive performance, however there was no significant difference in risk between the two older age groups (21-29 vs. 30 or older). Some other studies in this field have examined the influence of parity on cognitive function and have predominantly reported non-significant associations (Smith et al., 1999; Dunkin et al., 2005; Rasgon et al., 2005), however no previous study has examined the potential association between age at childbirth and later life cognition. Additional research is required to validate and further explain the association between young mothers and poor cognitive function.

\section{Hormone Therapy}

In the multivariate analysis, current HT was significantly associated with better visual memory. This result is supported by previous studies which have demonstrated that women using HT performed better and with 
fewer errors on tasks of Weschler Memory Scale Visual Reproduction test (Smith et al., 2001) and the BVRT (Resnick et al., 1997). In contrast, the majority of previous studies of visual memory failed to find a significant association with HT use (Hogervorst et al., 2002a). Our results also suggest that current HT may have a beneficial effect on word recall, executive function and psychomotor speed, with odds ratios all in the range of 0.5 to 0.6 , although these associations failed to reach the $5 \%$ significance level. Past treatment was not associated with cognition function, suggesting any positive effect is removed once treatment is stopped, however other studies have indicated that HT can have long-lasting protective effects (Zandi et al., 2002).

There is currently no clear consensus concerning the benefits of postmenopausal HT on later life cognitive function. Some observational studies and short-term RCTs indicate that HT could be beneficial for some areas of cognitive function (Yaffe et al., 1998 ; Leblanc et al., 2001; Hogervorst et al., 2002a; Shaywitz et al., 2003; Rasgon et al., 2005; Resnick et al., 2006) however, other studies have reported no change in cognitive performance (Grady et al., 2002; Kang et al., 2004; Schiff et al., 2005; Kok et al., 2006; Pefanco et al., 2007). In addition, the WHIMS actually found an adverse effect on global cognitive function (Rapp et al., 2003; Espeland et al., 2004) and verbal memory (Resnick et al., 2006). These inconsistencies across studies are thought to be the consequence of variations related directly to the treatments (types, modes of administration, timing and duration of use), as well as differences between study populations (ages, sizes and health status) and methodology used. As the results of our study and others suggest, variations across cognitive domains would also be expected (Leblanc et al., 2001; MacLennan et al., 2006; Resnick et al., 2006). In fact, where positive associations between HT and cognitive function in older women have been reported in the literature, these have predominantly being improvements in verbal memory (Maki, 2006), which just failed to reach significance in our analysis. In addition, it may be that surgically menopausal women would benefit most from HT (Hogervorst et al., 2002a; Henderson and Sherwin, 2007), although in our study we found no difference in cognitive performance between natural or surgically menopausal women, and we also found no interaction between HT use and type of menopause. These findings are limited however, because we did not consider the age of the women at surgical menopause or the duration of HT use following surgery.

\section{Decline in cognitive function over time}


Lifetime hormonal factors were not significantly associated with cognitive decline over follow-up. As significant associations were identified in the cross-sectional analysis, this suggests that reproductive events could potentially influence cognitive function at an earlier age, but have minimal further impact on cognitive decline in later life. This would not be surprising given that some of the exposures, such as age at first menses, occur very early on in a woman's reproductive life. Nevertheless, of the scant longitudinal studies which have investigated the association between events across the female reproductive life and cognitive decline in older age, some significant associations have been reported. McLay and colleagues followed 361 postmenopausal women for a median of 12.8 years and reported that women who were nulliparous or underwent menopause at a later age, have a significantly reduced risk of decline in global cognitive function (MMSE), while there was no association with oral contraceptive use or surgical menopause (McLay et al., 2003). The longer follow-up time may explain the presence of significant associations which were not found in our analysis; however both studies have identified similar hormonal events, once again suggesting an influence of endogenous estrogen exposure on cognitive function.

There has also been one report, based on data collected from the Rotterdam study, that a longer reproductive period was actually associated with an increased incidence of dementia in naturally postmenopausal women (Geerlings et al., 2001). In fact, the positive association they reported was only apparent among women who were carriers of the ApoE- $\varepsilon 4$ gene, which is known to increase susceptibility to developing AD. In our logistic models of poor cognitive performance, we found no significant interactions between reproductive period or age at menopause, and ApoE- $\varepsilon 4$ status (data not shown).

We failed to find a significant association between current HT use and cognitive decline or dementia, which is supported by other longitudinal studies of postmenopausal women (Geerlings et al., 2001; Petitti et al., 2008). With a longer follow-up period however, we would expect more substantial decline in cognitive function among the participants, and therefore, the influence of HT may become more apparent. In the current analysis, many women actually performed better on the cognitive tasks at follow-up, more likely due to a learning effect. For several of the tasks the median change over time was actually positive (improved scores), while for others there was a median of no change in test scores. With regards to the MMSE and the word list of Dubois, it is also likely that there is a ceiling effect because both of these tests are probably not sensitive to minor cognitive changes, especially in non-demented people. 
Data in the literature indicates that the effects of HT on cognitive function are most likely influenced by the type of hormone treatment and the mode of administration (MacLennan et al., 2006). The size of our study did not allow us to distinguish between transdermal and oral preparations or between estrogens versus combined estrogen-progesterone treatments. It is important to note, however, that the women in our study used predominantly transdermal estradiol (with or without progesterone), with less than $20 \%$ taking oral estradiol. In addition, in contrast to the North American studies, none of the women were taking compounds such as conjugated-equine estrogen, which may also help explain some of the beneficial effects we found in the cross-sectional analysis, which have not been reported previously.

Future analysis using a larger population-based longitudinal dataset will enable us to examine more closely the effect of hormone treatment on cognitive function. In addition to the types of preparations and mode of administration, we will also focus on the timing of initiation of treatment and duration of use in relation to specific periods such as the menopause. Indeed, our cross-sectional analysis suggested a trend for long-term HT use ( $\geq 10$ years) having a more beneficial effect on certain area of cognitive function than short-term use (Supplementary Table 1).

\section{Limitations \& Strengths}

The principal limitation of this study is the retrospective nature of the data concerning lifetime hormonal exposure, even if we have excluded women with possible dementia at baseline to try and minimise inaccuracies which would bias the results. While age at first menses is an event that occurred several decades ago for the women in this study, research suggests that up to $90 \%$ of women could accurately report their age at menarche within a 1-year error margin (Bean et al., 1979). In addition, we have previously observed a high level of reproducibility in terms of their age at menopause and type of menopause, between the responses at baseline and at the follow-up interviews (Ryan et al., 2008a). We would also expect that other characteristics such as age at the birth of a woman's first child and her number of children would be remembered with a high degree of accuracy. Therefore, although women with low cognitive performance may have more trouble recalling past events in their reproductive life, it seems that this differential recall would not have substantially influenced the results of our study. Finally, it is possible that there are other unknown factors which may confound the associations reported here or that by examining multiple associations, we have increased the probability of a type 1 error. 
We need to also consider bias due to the exclusion of some of the participants that were initially recruited to the ESPRIT study. However there was no significant difference in terms of the hormone-related characteristics between women in this analysis and the entire cohort. In addition, women with more extreme cognitive problems (i.e. probable or possible dementia) were excluded from this analysis, and therefore we may have had reduced power to detect significant associations if they were present.

Our study does have a number of strengths. The analysis was based on a relatively large populationbased sample of women, and therefore the results may be relevant to the broader community of older women. This study brings some important new findings to the field of hormone exposure and later life cognitive function. Both cross-sectional and longitudinal analyses were undertaken, a number of reproductive characteristics were investigated and a range of cognitive domains assessed. In addition, we have taken into account a wide range of competing causes of cognitive dysfunction in the elderly, by controlling for socio-demographic, health and lifestyle covariates and thus limiting any potential confounding.

\section{Conclusion}

The majority of studies investigating the influence of estrogen on cognitive function have tended to focus on the effects of HT taken at or after the menopause, at a time where neurodegeneration may have already started. They have seldom considered the heterogeneity amongst women in terms of their hormonal exposure across the reproductive lifetime and how this could affect later-life cognitive function. Our study extends previous findings which suggest that in addition to current HT, there are a small number of reproductive factors which reflect an increased endogenous estrogen exposure (notable timing of first menses, length of the reproductive period and age at the birth of the first child), that are also associated with better cognitive functioning, notably visual memory, psychomotor speed, verbal fluency and global cognitive function. We did not however observe any beneficial or detrimental effects on dementia incidence over the four year follow-up, but this may result from a lack of statistical power as only 27 of the 996 women developed dementia during the study period. As this study is ongoing, it will be possible to re-examine this question over a longer follow-up period. Further studies will also be required to determine whether hormone exposure across the lifetime could influence how a woman responds to HT in later life. 


\section{REFERENCES}

American Psychiatric Association 1994. Diagnostic and Statistical Manual of Mental Disorders (DSM-IV). American Psychiatric Association, American Psychiatric Association

Ancelin, M.L., Artero, S., Portet, F., Dupuy, A.M., Touchon, J., Ritchie, K., 2006. Non-degenerative mild cognitive impairment in elderly people and use of anticholinergic drugs: longitudinal cohort study. Bmj. 332, 455-9.

Ancelin, M.L., Ritchie, K., 2005. Lifelong endocrine fluctuations and related cognitive disorders. Current Pharm Design. 11, 4229-52.

Andersen, K., Launer, L.J., Dewey, M.E., Letenneur, L., Ott, A., Copeland, J.R., Dartigues, J.F., Kraghsorensen, P., Baldereschi, M., Brayne, C., Lobo, A., Martinez-lage, J.M., Stijnen, T., Hofman, A., 1999. Gender differences in the incidence of AD and vascular dementia: The EURODEM Studies. EURODEM Incidence Research Group. Neurology. 53, 1992-97.

Apter, D., Reinila, M., Vihko, R., 1989. Some endocrine characteristics of early menarche, a risk factor for breast cancer, are preserved into adulthood. Int J Cancer. 44, 783-7.

Apter, D., Vihko, R., 1983. Early menarche, a risk factor for breast cancer, indicates early onset of ovulatory cycles. J Clin Endocrinol Metab. 57, 82-6.

Bean, J.A., Leeper, J.D., Wallace, R.B., Sherman, B.M., Jagger, H., 1979. Variations in the reporting of menstrual histories. Am J Epidemiol. 109, 181-5.

Beh1, C., 2002. Oestrogen as a neuroprotective hormone. Nat Rev Neurosci. 3, 433-42.

Benton, A.L. 1965 Manuel pour l'application du test de retention visuelle. Applications cliniques et experimentales. In. Centre de Psychologie Appliquee., Paris

Bernstein, L., Depue, R.H., Ross, R.K., Judd, H.L., Pike, M.C., Henderson, B.E., 1986. Higher maternal levels of free estradiol in first compared to second pregnancy: early gestational differences. J Natl Cancer Inst. 76, 1035-9.

Bernstein, L., Pike, M.C., Ross, R.K., Judd, H.L., Brown, J.B., Henderson, B.E., 1985. Estrogen and sex hormone-binding globulin levels in nulliparous and parous women. J Natl Cancer Inst. 74, 741-5.

Bernstein, L., Ross, R.K., 1993. Endogenous hormones and breast cancer risk. Epidemiol Rev. 15, 48-65.

Britt, K., Ashworth, A., Smalley, M., 2007. Pregnancy and the risk of breast cancer. Endocr Relat Cancer. $14,907-33$.

Clavel-Chapelon, F., 2002. Cumulative number of menstrual cycles and breast cancer risk: results from the E3N cohort study of French women. Cancer Causes Control. 13, 831-8.

Colucci, M., Cammarata, S., Assini, A., Croce, R., Clerici, F., Novello, C., Mazzella, L., Dagnino, N., Mariani, C., Tanganelli, P., 2006. The number of pregnancies is a risk factor for Alzheimer's disease. Eur J Neurol. 13, 1374-7.

Commenges, D., Letenneur, L., Joly, P., Alioum, A., Dartigues, J.F., 1998. Modelling age-specific risk: application to dementia. Stat Med. 17, 1973-88.

Dorgan, J.F., Reichman, M.E., Judd, J.T., Brown, C., Longcope, C., Schatzkin, A., Campbell, W.S., Franz, C., Kahle, L., Taylor, P.R., 1995. Relationships of age and reproductive characteristics with plasma estrogens and androgens in premenopausal women. Cancer Epidemiol Biomarkers Prev. 4, 381-6.

Dubois, B., 2001. L'epreuve des cinq mots. Neurology Psychiatrie Geriatr. 1, 40-42.

Dufouil, C., Richard, F., Fievet, N., Dartigues, J.F., Ritchie, K., Tzourio, C., Amouyel, P., Alperovitch, A., 2005. APOE genotype, cholesterol level, lipid-lowering treatment and dementia. Neurology. 64, 1531-38.

Dunkin, J., Rasgon, N., Wagner-Steh, K., David, S., Altshuler, L., Rapkin, A., 2005. Reproductive events modify the effects of estrogen replacement therapy on cognition in healthy postmenopausal women. Psychoneuroendocrinology. 30, 284-96.

Espeland, M.A., Rapp, S.R., Shumaker, S.A., Brunner, R., Manson, J.E., Sherwin, B.B., Hsia, J., Margolis, K.L., Hogan, P.E., Wallace, R., Dailey, M., Freeman, R., Hays, J., 2004. Conjugated equine estrogens and global cognitive function in postmenopausal women: Women's Health Initiative Memory Study. Jama. 291, 2959-68.

Folstein, M.F., Folstein, S.E., McHugh, P.R., 1975. "Mini-mental state". A practical method for grading the cognitive state of patients for the clinician. J Psychiatr Res. 12, 189-98.

Gao, S., Hendrie, H.C., Hall, K.S., Hui, S., 1998. The relationships between age, sex, and the incidence of dementia and Alzheimer disease: a meta-analysis. Arch Gen Psychiatry. 55, 809-15. 
Geerlings, M.I., Ruitenberg, A., Witteman, J.C., Van Swieten, J.C., Hofman, A., Van Duijn, C.M., Breteler, M.M., Launer, L.J., 2001. Reproductive period and risk of dementia in postmenopausal women. Jama. 285, 1475-81.

Grady, D., Yaffe, K., Kristof, M., Lin, F., Richards, C., Barrett-Connor, E., 2002. Effect of postmenopausal hormone therapy on cognitive function: the Heart and Estrogen/progestin Replacement Study. Am J Med. 113, 543-8.

Grodstein, F., Chen, J., Pollen, D.A., Albert, M.S., Wilson, R.S., Folstein, M.F., Evans, D.A., Stampfer, M.J., 2000. Postmenopausal hormone therapy and cognitive function in healthy older women. J Am Geriatr Soc. 48, 746-52.

Henderson, V.W., Guthrie, J.R., Dudley, E.C., Burger, H.G., Dennerstein, L., 2003. Estrogen exposures and memory at midlife: a population-based study of women. Neurology. 60, 1369-71.

Henderson, V.W., Sherwin, B.B., 2007. Surgical versus natural menopause: cognitive issues. Menopause. $14,572-9$.

Hogervorst, E., Yaffe, K., Richards, M., Huppert, F., 2002a. Hormone replacement therapy for cognitive function in postmenopausal women. Cochrane. Database. Syst. Rev. CD0031.

Hogervorst, E., Yaffe, K., Richards, M., Huppert, F., 2002b. Hormone replacement therapy to maintain cognitive function in women with dementia (Cochrane Review). Cochrane. Database. Syst. Rev. CD0037.

Isaacs, B., Kennie, A.T., 1973. The Set Test as an aid to the detection of dementia in old people. British Journal of Psychiatry. 45, 957-62.

Kalache, A., Maguire, A., Thompson, S.G., 1993. Age at last full-term pregnancy and risk of breast cancer. Lancet. 341, 33-6.

Kang, J.H., Weuve, J., Grodstein, F., 2004. Postmenopausal hormone therapy and risk of cognitive decline in community-dwelling aging women. Neurology. 63, 101-7.

Katz, S., Ford, A.B., Moskowitz, R.W., Jaffee, M.W., 1963. Studies of illness in the aged. The index of ADL: a standardized measure of biological and psychosocial function. JAMA. 195, 94-99.

Kok, H.S., Kuh, D., Cooper, R., van der Schouw, Y.T., Grobbee, D.E., Wadsworth, M.E., Richards, M., 2006. Cognitive function across the life course and the menopausal transition in a British birth cohort. Menopause. 13, 19-27.

Kuh, D., Butterworth, S., Kok, H., Richards, M., Hardy, R., Wadsworth, M.E., Leon, D.A., 2005. Childhood cognitive ability and age at menopause: evidence from two cohort studies. Menopause. 12, 475-82.

Lawton, M.P., Brody, E.M., 1969. Assessment of older people: self-maintaining and instrumental activities of daily living. Gerontologist. 9, 179-86.

Leblanc, E.S., Janowsky, J., Chan, B.K., Nelson, H.D., 2001. Hormone replacement therapy and cognition: systematic review and meta-analysis. Jama. 285, 1489-99.

Lebrun, C.E., van der Schouw, Y.T., de Jong, F.H., Pols, H.A., Grobbee, D.E., Lamberts, S.W., 2005. Endogenous oestrogens are related to cognition in healthy elderly women. Clin Endocrinol (Oxf). $63,50-5$.

Low, L.F., Anstey, K.J., Jorm, A.F., Rodgers, B., Christensen, H., 2005. Reproductive period and cognitive function in a representative sample of naturally postmenopausal women aged 60-64 years. Climacteric. 8, 380-9.

MacLennan, A.H., Henderson, V.W., Paine, B.J., Mathias, J., Ramsay, E.N., Ryan, P., Stocks, N.P., Taylor, A.W., 2006. Hormone therapy, timing of initiation, and cognition in women aged older than 60 years: the REMEMBER pilot study. Menopause. 13, 28-36.

MacMahon, B., Trichopoulos, D., Brown, J., Andersen, A.P., Aoki, K., Cole, P., deWaard, F., Kauraniemi, T., Morgan, R.W., Purde, M., Ravnihar, B., Stromby, N., Westlund, K., Woo, N.C., 1982. Age at menarche, probability of ovulation and breast cancer risk. Int J Cancer. 29, 13-6.

Maki, P., Hogervorst, E., 2003. The menopause and HRT. HRT and cognitive decline. Best Pract Res Clin Endocrinol Metab. 17, 105-22.

Maki, P.M., 2006. Hormone therapy and cognitive function: is there a critical period for benefit? Neuroscience. 138, 1027-30.

McLay, R.N., Maki, P.M., Lyketsos, C.G., 2003. Nulliparity and late menopause are associated with decreased cognitive decline. J Neuropsychiatry Clin Neurosci. 15, 161-7.

Musey, V.C., Collins, D.C., Brogan, D.R., Santos, V.R., Musey, P.I., Martino-Saltzman, D., Preedy, J.R., 1987. Long term effects of a first pregnancy on the hormonal environment: estrogens and androgens. J Clin Endocrinol Metab. 64, 111-8. 
Nappi, R.E., Sinforiani, E., Mauri, M., Bono, G., Polatti, F., Nappi, G., 1999. Memory functioning at menopause: impact of age in ovariectomized women. Gynecol Obstet Invest. 47, $29-36$.

National Heart, L., and Blood Institute 1998 Clinical Guidelines on the Identification, Evaluation, and Treatment of Overweight and Obesity in Adults The Evidence Report. In: Publication N (ed)

Paganini-Hill, A., Henderson, V.W., 1994. Estrogen deficiency and risk of Alzheimer's disease in women. Am. J. Epidemiol. 140, 256-61.

Pefanco, M.A., Kenny, A.M., Kaplan, R.F., Kuchel, G., Walsh, S., Kleppinger, A., Prestwood, K., 2007. The effect of 3-year treatment with $0.25 \mathrm{mg} /$ day of micronized 17 beta-estradiol on cognitive function in older postmenopausal women. J Am Geriatr Soc. 55, 426-31.

Petitti, D.B., Crooks, V.C., Chiu, V., Buckwalter, J.G., Chui, H.C., 2008. Incidence of dementia in long-term hormone users. Am J Epidemiol. 167, 692-700.

Ptok, U., Barkow, K., Heun, R., 2002. Fertility and number of children in patients with Alzheimer's disease. Arch Women Ment Health. 5, 83-6.

Radloff, L., 1977. The CES-D scale: a self-report depression scale for research in the general population. Appl Psychol Measurement. 1, 385-401.

Rapp, S.R., Espeland, M.A., Shumaker, S.A., Henderson, V.W., Brunner, R.L., Manson, J.E., Gass, M.L., Stefanick, M.L., Lane, D.S., Hays, J., Johnson, K.C., Coker, L.H., Dailey, M., Bowen, D., 2003. Effect of estrogen plus progestin on global cognitive function in postmenopausal women: the Women's Health Initiative Memory Study: a randomized controlled trial. Jama. 289, 2663-72.

Rasgon, N.L., Magnusson, C., Johansson, A.L., Pedersen, N.L., Elman, S., Gatz, M., 2005. Endogenous and exogenous hormone exposure and risk of cognitive impairment in Swedish twins: a preliminary study. Psychoneuroendocrinology. 30, 558-67.

Reitan, R.M., 1965. Validity of the Trail Making Test as an indicator of organic brain damage. Percept Motor Skills. 8, 271-76.

Resnick, S.M., Maki, P.M., Rapp, S.R., Espeland, M.A., Brunner, R., Coker, L.H., Granek, I.A., Hogan, P., Ockene, J.K., Shumaker, S.A., 2006. Effects of combination estrogen plus progestin hormone treatment on cognition and affect. J Clin Endocrinol Metab. 91, 1802-10.

Resnick, S.M., Metter, E.J., Zonderman, A.B., 1997. Estrogen replacement therapy and longitudinal decline in visual memory. A possible protective effect? Neurology. 49, 1491-97.

Ritchie, K., Artero, S., Beluche, I., Ancelin, M.L., Mann, A., Dupuy, A.M., Malafosse, A., Boulenger, J.P., 2004. Prevalence of DSM-IV psychiatric disorder in the French elderly population. Br J Psychiatry. $184,147-52$.

Ritchie, K., Carriere, I., de Mendonca, A., Portet, F., Dartigues, J.F., Rouaud, O., Barberger-Gateau, P., Ancelin, M.L., 2007. The neuroprotective effects of caffeine: a prospective population study (the Three City Study). Neurology. 69, 536-45.

Ryan, J., Carriere, I., Scali, J., Ritchie, K., Ancelin, M.L., 2008a. Lifetime hormonal factors may predict latelife depression in women. Int Psychogeriatr. June 5: 1-16 [Epub ahead of print].

Ryan, J., Scali, J., Carriere, I., Ritchie, K., Ancelin, M.L., 2008b. Hormonal treatment, mild cognitive impairment and Alzheimer's disease. Int Psychogeriatr. 20, 47-56.

Schiff, R., Bulpitt, C.J., Wesnes, K.A., Rajkumar, C., 2005. Short-term transdermal estradiol therapy, cognition and depressive symptoms in healthy older women. A randomised placebo controlled pilot cross-over study. Psychoneuroendocrinology. 30, 309-15.

Shaywitz, S.E., Naftolin, F., Zelterman, D., Marchione, K.E., Holahan, J.M., Palter, S.F., Shaywitz, B.A., 2003. Better oral reading and short-term memory in midlife, postmenopausal women taking estrogen. Menopause. 10, 420-6.

Sherwin, B.B., 2003. Estrogen and cognitive functioning in women. Endocr Rev. 24, 133-51.

Sherwin, B.B., Henry, J.F., 2008. Brain aging modulates the neuroprotective effects of estrogen on selective aspects of cognition in women: a critical review. Front Neuroendocrinol. 29, 88-113.

Shumaker, S.A., Legault, C., Thal, L., Wallace, R.B., Ockene, J.K., Hendrix, S.L., Jones, B.N., 3rd, Assaf, A.R., Jackson, R.D., Kotchen, J.M., Wassertheil-Smoller, S., Wactawski-Wende, J., 2003. Estrogen plus progestin and the incidence of dementia and mild cognitive impairment in postmenopausal women: the Women's Health Initiative Memory Study: a randomized controlled trial. Jama. 289, 2651-62.

Smith, C.A., Mccleary, C.A., Murdock, G.A., Wilshire, T.W., Buckwalter, D.K., Bretsky, P., Marmol, L., Gorsuch, R.L., Buckwalter, J.G., 1999. Lifelong estrogen exposure and cognitive performance in elderly women. Brain. Cogn. 39, 203-18. 
Smith, Y.R., Giordani, B., Lajiness-O'Neill, R., Zubieta, J.K., 2001. Long-term estrogen replacement is associated with improved nonverbal memory and attentional measures in postmenopausal women. Fertil Steril. 76, 1101-7.

Trapido, E.J., 1983. Age at first birth, parity, and breast cancer risk. Cancer. 51, 946-8.

WHO. 2000. World Health Organising Collaborating Centre for Drug Statistics Methodology. Guidelines for ATC classification and DDD assignment.

Yaffe, K., Sawaya, G., Lieberburg, I., Grady, D., 1998. Estrogen therapy in postmenopausal women: effects on cognitive function and dementia. Jama. 279, 688-95.

Zandi, P.P., Carlson, M.C., Plassman, B.L., Welsh-Bohmer, K.A., Mayer, L.S., Steffens, D.C., Breitner, J.C., 2002. Hormone replacement therapy and incidence of Alzheimer disease in older women: the Cache County Study. Jama. 288, 2123-29. 